\title{
Métodos de Pontos Interiores Aplicados à Basis Pursuit
}

\author{
Aurelio Ribeiro Leite de Oliveira, $\quad$ Paula Aparecida Kikuchi, \\ Departamento de Matemática Aplicada, IMECC, Unicamp, \\ 13083-859, Campinas, SP \\ E-mail: aurelio@ime.unicamp.br, paulapkikuchi@gmail.com,

\section{Daniela Renata Cantane} \\ Departamento de Bioestatística, Instituto de Biociências, Unesp \\ Campus de Botucatu \\ 18618-970, Botucatu, SP \\ E-mail: dcantane@ibb.unesp.br.
}

\begin{abstract}
Resumo: Vários são os métodos propostos para reconstrução de sinal. Nosso enfoque é o método Basis Pursuit. Trabalhando com dicionários overcomplete, são inúmeras as combinações possiveis para a representação do sinal e Basis Pursuit encontra a mais esparsa. Veremos que podemos reescrever o problema em questão como um problema de programação linear. Apresentaremos um método já existente para a resolução deste problema, o Método Primal-Dual Barreira Logarítmica. Vamos aplicar o Método Barreira Logarítmica, e buscando maior eficiência, iremos incluir a direção afim-escala, a direção de centragem e a direção de correção no mesmo método, obtendo o Método Primal-Dual Barreira Logarítmica Preditor-Corretor. Resultados computacionais com problemas reais comprovam a eficiência do método proposto.
\end{abstract}

Palavras-chave: representação de sinais, esparsidade, programação linear, método de barreira, método preditor-corretor

\section{Introdução}

Dado um sinal, podemos decompô-lo como combinação linear de certos elementos [1], para tanto, vamos definir certos conceitos.

Definimos dicionário como uma coleção de waveforms parametrizadas $D=\left(\phi_{\gamma}: \gamma \in \Gamma\right)$, sendo waveforms $\phi_{\gamma}$ sinais discretos de tempo chamados átomos, que nesse trabalho será visto como um vetor pertencente a $\mathbb{R}^{n}$. Representação de sinais por meio de dicionários é uma alternativa que surge, além das representações usuais que utilizam superposição de senóides, como mencionado em [4].

Consideramos um sinal $s$ sendo um vetor pertencente a $\mathbb{R}^{n}$, sua decomposição em um dicionário é dada por $s=\sum_{\gamma \in \Gamma} \alpha_{\gamma} \phi_{\gamma}$. Considere que temos um dicionário discreto de $p$ waveforms e uma matriz $\Phi$ cujas colunas correspondem às $p$ waveforms $(\Phi: n \times p)$, podemos reescrever $s$ como $\Phi \alpha=s$, onde $\alpha=\left(\alpha_{\gamma}\right)$ é o vetor dos coeficientes da representação anterior.

Veja que, se as waveforms são linearmente independentes, teremos uma matriz $\Phi$ não singular, sendo que se as waveforms forem ortonormais $\Phi^{-1}=\Phi^{T}$.

Dicionários são definidos como completos quando possuem $n$ átomos, overcomplete quando possuem mais que $n$ átomos, ou undercomplete quando possuem menos de $n$ átomos.

Note que se trabalhamos com um dicionário completo, $s$ terá uma representação única. Já quando fazemos uso de dicionários overcomplete, sua representação não será única (teremos átomos que são combinações lineares de outros), permitindo que escolhamos a que nos for mais 
conveniente. Como um dos objetivos que queremos alcançar nas representações de sinais é esparsidade (obter o menor número de coeficientes significativos), já podemos ter em mente que uma boa ideia seria minimizar $\sum_{\gamma \in \Gamma}\left|\alpha_{\gamma}\right|$.

Não são poucos os métodos propostos para representar sinais em dicionários overcomplete. Alguns exemplos, descritos em [3] são: método de frames (MOF), que escolhe a representação cujos coeficientes tenham a norma 2 mínima, matching pursuit (MP), the best orthogonal basis (BOB) e basis pursuit (BP). Diferentemente do método de frames, BP irá escolher a representação cujos coeficientes tenham a norma 1 mínima. Chamamos essa decomposição do sinal de superposição "ótima" de elementos do dicionário. Chen, Donoho e Saunders [4] mostram várias vantagens de $\mathrm{BP}$ sobre $\mathrm{MOF}, \mathrm{MP}$ e $\mathrm{BOB}$, dentre elas, esparsidade.

Note que BP baseia-se na resolução do seguinte problema:

$$
\begin{array}{ll}
\operatorname{minimizar} & \|\alpha\|_{1} \\
\text { sujeito a } & \Phi \alpha=s
\end{array} .
$$

Sendo que podemos reformulá-lo por meio de um programa linear na forma padrão [8].

Resolveremos o problema linear na forma padrão, equivalente a (1), por meio do método de pontos interiores, que será o enfoque desse trabalho.

\section{Métodos de Pontos Interiores Aplicados à Basis Pursuit}

Como vimos, o problema BP pode ser reescrito como um problema linear. Nosso enfoque será trabalhar com este problema aplicando Métodos de Pontos Interiores $[2,6,11,12,13]$.

Vamos analisar o Método Primal-Dual Barreira Logarítmica proposto em [3] para o problema BP. No programa referente a tal método (BP_Interior), que faz parte de um pacote denominado Atomizer, implementado em Matlab e que pode ser encontrado em [5], vamos fazer uso do método proposto por Gill, Murray e Saunders [6], reescrevendo o problema como o seguinte programa linear perturbado:

$$
\begin{array}{cl}
\operatorname{minimizar} & c^{T} x+\frac{1}{2}\|\gamma x\|_{2}^{2}+\frac{1}{2}\|p\|_{2}^{2} \\
\text { sujeito a } & A x+\delta p=b \\
& x \geq 0
\end{array}
$$

onde $\gamma$ e $\delta$ são parâmetros de perturbação pequenos, da ordem de $\left(10^{-4}\right)$; e resolvendo-o assim como feito em [3].

Associamos ao problema linear perturbado (2) o subproblema barreira logarítmica:

$$
\begin{array}{ll}
\text { minimizar } & c^{T} x+\frac{1}{2}\|\gamma x\|_{2}^{2}+\frac{1}{2}\|p\|_{2}^{2}-\mu \sum_{i=1}^{m} \ln \left(x_{i}\right) . \\
\text { sujeito a } & A x+\delta p=b
\end{array}
$$

A restrição de desigualdade $x \geq 0$ fica implícita. Como $\mu \rightarrow 0$ a solução converge para a solução do problema linear perturbado.

Definimos o lagrangeano como:

$$
L(x, y, p)=c^{T} x+\frac{1}{2}\|\gamma x\|_{2}^{2}+\frac{1}{2}\|p\|_{2}^{2}-\mu \sum_{i=1}^{m} \ln \left(x_{i}\right)+y^{T}(b-A x-\delta p) .
$$

Agora vamos calcular as condições necessária de primeira ordem do problema de minimização da função Lagrangeana:

$$
\operatorname{minimizar} c^{T} x+\frac{1}{2}\|\gamma x\|_{2}^{2}+\frac{1}{2}\|p\|_{2}^{2}-\mu \sum_{i=1}^{m} \ln \left(x_{i}\right)+y^{T}(b-A x-\delta p),
$$

obtendo, 


$$
-\nabla_{x} L=A^{T} y-\gamma^{2} x-c+\mu X^{-1} e=0,
$$

onde $e$ é o vetor de dimensão apropriada, cujos elementos são compostos por 1 .

No artigo de McShane, Monma e Shanno [10] vemos que quando $\mu$ do problema primal e dual são iguais, temos que $z=\mu X^{-1} e$. Assim as condições necessárias de primeira ordem são dadas por:

$$
\begin{aligned}
& -\nabla_{x} L=A^{T} y-\gamma^{2} x-c+z=0 \\
& -\nabla_{p} L=\delta y-p=0 \Rightarrow p=\delta y \\
& -\nabla_{y} L=A x+\delta p-b=A x+\delta^{2} y-b=0
\end{aligned}
$$

e por [10] novamente:

$$
Z X e-\mu e=0
$$

Rearranjando as expressões, obtemos

$$
F(x, y, z)=\left(\begin{array}{c}
A^{T} y-\gamma^{2} x-c+z \\
A x+\delta^{2} y-b \\
Z X e-\mu e
\end{array}\right)=0
$$

onde $z$ é um vetor dual, $X$ e $Z$ são as matrizes diagonais formadas pelos elementos dos vetores $x$ e $z$ respectivamente.

Aplicamos o Método de Newton a fim de encontrar a solução do sistema linear acima. As direções de Newton $(\Delta x, \Delta y, \Delta z)$ devem satisfazer o sitema:

$$
\begin{aligned}
& \left(A D A^{T}+\delta^{2} I\right) \Delta y=r-A D\left(X^{-1} v-t\right), \\
& \Delta x=D A^{T} \Delta y+D\left(X^{-1} v-t\right), \\
& \Delta z=X^{-1} v-X^{-1} Z \Delta x .
\end{aligned}
$$

sendo $D=\left(X^{-1} Z+\gamma^{2} I\right)^{-1}$. Para a escolha do comprimento do passo $\left(\rho_{p}, \rho_{d}\right)$, vamos sempre escolher o maior possível, contanto que o ponto $x^{(k+1)}$ e $z^{(k+1)}$ sejam pontos interiores. A cada passo de Newton vamos decrescer o parâmetro de barreira $\mu$ monotonicamente e mais rapidamente se largos passos são tomados:

$$
\mu \leftarrow\left(1-\min \left(\rho_{p}, \rho_{d}, 0,99\right)\right) \bar{\mu} .
$$

sendo $\bar{\mu}$ o valor de $\mu$ do passo anterior.

Resolvemos (3) para $\Delta y$ pelo Método dos Gradientes Conjugados [7].

O método converge quando $\left\|b-A x-\delta^{2} y\right\|_{2},\left\|c+\gamma^{2} x-z-A^{T} y\right\|_{2}$ e $z^{T} x$ são suficientemente pequenos.

O programa BP_Interior desenvolvido por Chen, contido em Atomizer [5], resolve o problema Basis Pursuit (1) seguindo o mesmo procedimento descrito no Método Primal-Dual Barreira Logarítmica.

Aplicamos o Método Barreira Logarítmica no problema Basis Pursuit (1), neste caso não teremos a condição de complementaridade, o que mudará o critério de convergência visto no método Primal-Dual Barreira Logarítmica, maiores detalhes encontram-se em [8]; e a fim de melhorar o Método Primal-Dual Barreira Logarítmica vamos introduzir as três componentes: Direção Afim-Escala, Direção de Centragem e Direção de Correção. Tendo em vista esses conceitos, e em busca de melhorar o desempenho de BP_Interior, fazemos as correções dos termos não lineares do método descrito anteriormente. Note que o termo não linear corresponde à 
condição de complementaridade. Assim, no primeiro passo consideraremos $v$ tal que $v=-Z x$, e no segundo passo $v=\mu e-Z x-\Delta Z \Delta X e$, onde $\Delta Z$ e $\Delta X$ são as matrizes diagonais cujos elementos são as entradas dos vetores $\Delta z$ e $\Delta x$ repectivamente, que foram obtidos no primeiro passo.

Considere o Método Barreira Logarítmica e o Método Primal-Dual Barreira Logarítmica Preditor-Corretor, como BP_BL e BP_InteriorPC, respectivamente. Sendo Df a diferença relativa do valor da função objetivo do passo atual com o passo anterior e MPC referente ao Método Preditor-Corretor. Apresentamos nas Tabelas (1) e (2) um resumo dos métodos.

Tabela 1: Resumo dos Métodos.

\begin{tabular}{|c|c|c|}
\hline Método & BP_Interior & BP_BL \\
\hline $\begin{array}{l}\text { Conjunto de } \\
\text { variáveis }\end{array}$ & $\mathrm{x}, \mathrm{y}$ e $\mathrm{z}$ & $\mathrm{x}$ e $\mathrm{y}$ \\
\hline Propriedades & $\begin{array}{l}\text { Com condição de } \\
\text { complementaridade } \\
\text { e sem direções de } \\
\text { correções do MPC }\end{array}$ & $\begin{array}{l}\text { Sem condição de } \\
\text { complementaridade } \\
\text { e sem direções de } \\
\text { correções do MPC }\end{array}$ \\
\hline $\begin{array}{c}\text { Critérios de } \\
\text { Parada }\end{array}$ & $\begin{array}{l}\text { Infactibilidade Primal: } \\
\frac{\left\|b-A x-\delta^{2} y\right\|_{2}}{1+\|x\|_{2}}<\text { Featol; } \\
\text { Infactibilidade do Dual: } \\
\frac{\left\|c+\gamma^{2} x-z-A^{T} y\right\|_{2}}{1+\|y\|_{2}}<\text { Featol; } \\
\quad \text { Gap de Dualidade: } \\
\frac{z^{T} x}{1+\|x\|_{2}}<\text { PDGapTol. }\end{array}$ & $\begin{array}{c}\text { Infactibilidade Primal: } \\
\frac{\left\|b-A x-\delta^{2} y\right\|_{2}}{1+\|x\|_{2}}<\text { Featol; } \\
\text { Infactibilidade do Dual: } \\
\frac{\left\|c+\gamma^{2} x-\mu X^{-1} e-A^{T} y\right\|_{2}}{1+\|y\|_{2}}<\text { Featol. } \\
\text { ou se: } \\
\text { Df é menor que } 10^{-4} \text { em mais de três iterações. }\end{array}$ \\
\hline
\end{tabular}

Tabela 2: Resumo dos Métodos.

\begin{tabular}{|c|c|}
\hline Método & BP_InteriorPC \\
\hline $\begin{array}{c}\text { Conjunto de } \\
\text { variáveis }\end{array}$ & $\mathrm{x}, \mathrm{y} \mathrm{e} \mathrm{z}$ \\
\hline Propriedades & $\begin{array}{c}\text { Com condição de } \\
\text { complementaridade } \\
\text { e com direções de } \\
\text { correções do MPC }\end{array}$ \\
\hline Critérios de & $\frac{\left\|b-A x-\delta^{2} y\right\|_{2}}{\text { Infactibilidade Primal: }}<$ Featol; \\
Parada & $\begin{array}{c}\text { Infactibilidade do Dual: } \\
\left\|c+\gamma^{2} x-z-A^{T} y\right\|_{2}\end{array}$ \\
& $\begin{array}{c}1+\|y\|_{2} \\
G a p \text { de Dualidade: } \\
z^{T} x\end{array}$ \\
\hline
\end{tabular}

Os resumos dos métodos podem ser encontrados em [8]. 


\section{Resultados Computacionais}

A seguir, apresentamos os resultados obtidos para o Método BP_Interior de Chen [3], para o Método Barreira Logarítmica e para o nosso método modificado de BP_Interior: Método Primal-Dual Barreira Logarítmica Preditor-Corretor, os quais serão chamados de BP_BL e BP_InteriorPC, respectivamente.

Os experimentos numéricos foram implementados em Matlab R2010a, com o sistema operacional Linux, distribuição Ubuntu 11.04, processador Intel ${ }^{\circledR}$ core i7 2600, $3.4 \mathrm{Ghz}, 4 \mathrm{~GB}$ de memória DDR3, RAM clock 1333Mhz.

Na Tabela 3 listamos os sinais utilizados, com seus respectivos parâmetros, que são números ou vetores linha, o nome do dicionário que vamos utilizar para a representação e o tamanho do problema.

Tabela 3: Dados do problema.

\begin{tabular}{|l|r|l|r|r|r|}
\hline \multicolumn{1}{|c|}{ Sinal } & $\begin{array}{c}\text { Tamanho do } \\
\text { Problema }\end{array}$ & Dicionário & par1 & par2 & par3 \\
\hline TwinSine-1 & 256 & DCT & 4 & 0 & 0 \\
\hline WernerSorrows & 1024 & CP & 6 & seno & 0 \\
\hline Carbon & 1024 & WP & 10 & qmf & 0 \\
\hline TwinSine-2 & 256 & DCT & 6 & 0 & 0 \\
\hline FM-Cosine & 1024 & CP & 10 & seno & 0 \\
\hline Gong & 1024 & CP & Deno & 0 \\
\hline Dynamic-0 & 256 & DCT e DIRAC & MekeList $(4,0)$ & 0 & 0 \\
\hline Dynamic-2 & 256 & DCT e DIRAC & MekeList $(4,0)$ & 0 & 0 \\
\hline MultiGong & 256 & MDC & 8 & 1 & 0 \\
\hline
\end{tabular}

As Tabelas 4 e 5 mostram os resultados obtidos para BP_Interior, BP_InteriorPC e BP_BL.

Na Tabela 4 temos os valores da função objetivo, ou seja, o mínimo valor de $|\alpha|_{1}$ em (1), e o tempo decorrido até a obtenção da convergência, sendo que FO correponde ao valor da função objetivo e o tempo é dado em segundos.

Tabela 4: Resultados computacionais para a função objetivo e tempo de processamento.

\begin{tabular}{|l|c|r|c|r|r|r|}
\hline \multirow{2}{*}{ Sinal } & \multicolumn{2}{|c|}{ BP_Interior } & \multicolumn{2}{c|}{ BP_InteriorPC } & \multicolumn{2}{c|}{ BP_BL } \\
\cline { 2 - 7 } & \multicolumn{1}{c}{$\boldsymbol{F O}$} & Tempo & \multicolumn{1}{c}{$\boldsymbol{F O}$} & $\boldsymbol{T e m p o}$ & \multicolumn{1}{c}{$\boldsymbol{O}$} & Tempo \\
\hline TwinSine-1 & $2,00933 \mathrm{e}+00$ & 0,1 & $2,00934 \mathrm{e}+00$ & 0,1 & $2,01365 \mathrm{e}+00$ & 0,8 \\
WernerSorrows & $5,07482 \mathrm{e}+02$ & 249,2 & $5,07587 \mathrm{e}+02$ & 130,0 & $5,17296 \mathrm{e}+02$ & 78,0 \\
Carbon & $6,00247 \mathrm{e}+00$ & 19,8 & $6,00003 \mathrm{e}+00$ & 19,6 & $6,01948 \mathrm{e}+00$ & 707,3 \\
TwinSine-2 & $2,01150 \mathrm{e}+00$ & 0,1 & $2,01108 \mathrm{e}+00$ & 0,1 & $2,01425 \mathrm{e}+00$ & 1,4 \\
FM-Cosine & $2,52872 \mathrm{e}+02$ & 237,3 & $2,52885 \mathrm{e}+02$ & 210,9 & $2,54593 \mathrm{e}+02$ & 102,5 \\
Gong & $4,73088 \mathrm{e}+00$ & 3501,3 & $4,73171 \mathrm{e}+00$ & 1924,5 & $4,73566 \mathrm{e}+00$ & 2507,2 \\
Dynamic-0 & $6,01964 \mathrm{e}+00$ & 0,3 & $6,01902 \mathrm{e}+00$ & 0,4 & $6,01029 \mathrm{e}+00$ & 3,8 \\
Dynamic-2 & $4,03672 \mathrm{e}+02$ & 0,4 & $4,02187 \mathrm{e}+02$ & 0,7 & $4,88308 \mathrm{e}+02$ & 28,7 \\
MultiGong & $2,43810 \mathrm{e}+01$ & 5,6 & $2,44009 \mathrm{e}+01$ & 7,2 & $2,54105 \mathrm{e}+01$ & 15,8 \\
\hline
\end{tabular}

Na Tabela 5 é apresentado o número de iterações realizadas por cada método, e o número de iterações realizadas pelo Método dos Gradientes Conjugados (ItGC).

A relação entre o número de iterações e o valor da função objetivo, obtidos nos métodos implementados, com os sinais da Tabela 3 podem ser encontrados em [8].

Nota-se que BP_Interior e BP_InteriorPC possuem desempenho computacional equivalente em relação ao valor da função objetivo. BP_BL não teve um resultado satisfatório em relação aos outros, obtendo um valor da função objetivo melhor apenas em Dynamic-0, mas tendo um 
Tabela 5: Número de iterações dos métodos.

\begin{tabular}{|c|c|c|c|c|c|c|}
\hline \multirow[b]{2}{*}{ Sinal } & \multicolumn{2}{|c|}{ BP_Interior } & \multicolumn{2}{|c|}{ BP_InteriorPC } & \multicolumn{2}{|c|}{ BP_BL } \\
\hline & It & $I t G C$ & It & $I t G C$ & $I t$ & ItGC \\
\hline TwinSine-1 & 11 & 59 & 9 & 90 & 24 & 1745 \\
\hline WernerSorrows & 18 & 20883 & 11 & 10850 & 91 & 6687 \\
\hline Carbon & 8 & 85 & 6 & 99 & 35 & 4722 \\
\hline TwinSine-2 & 9 & 43 & 9 & 97 & 24 & 2950 \\
\hline FM-Cosine & 17 & 20098 & 12 & 17891 & 67 & 8862 \\
\hline Gong & 21 & 11510 & 19 & 21681 & 29 & 15377 \\
\hline Dynamic-0 & 7 & 40 & 5 & 54 & 24 & 3910 \\
\hline Dynamic-2 & 7 & 52 & 7 & 91 & 220 & 29022 \\
\hline MultiGong & 19 & 853 & 15 & 1097 & 57 & 11088 \\
\hline
\end{tabular}

tempo computacional bem maior. A diferença dos valores alcançados para a função objetivo deve-se ao fato do critério de convergência ser diferente para BP_BL.

Os tempos de convergência para BP_Interior, BP_InteriorPC foram similares, sendo as diferenças mais significativas obtidas para o sinal WernerSorrows, onde BP_Interior obteve 249, 2 segundos para a convergência, praticamente o dobro de tempo que BP_InteriorPC (130, 0 segundos); e para o sinal Gong, obtendo tempos bem distintos para os dois métodos, para BP_Interior obtemos 1924, 5 segundos para a convergência e para BP_InteriorPC 3501, 3 segundos. O tempo obtido por BP_BL foi menor que dos outros métodos apenas para WernerSorrows e FM-Cosine, contudo o valor da função objetivo obtida, não foi satisfatória.

Quando o número de iterações realizadas por BP_InteriorPC não foi menor que o número realizado por BP_Interior, essa mostrou-se igual.

Em relação ao Método dos Gradientes Conjugados podemos notar pelos resultados obtidos que o número de iterações do Método dos Gradientes Conjugados aumenta constantemente. Isso ocorre porque a solução inicial está próxima do centro da região factível. Assim, nas iterações iniciais, o sistema de equações (3) é bem condicionado e o método converge rapidamente. Mas, como $x^{T} z$ converge para $0, z / x$ convergirá para infinito ou 0 . Assim, a matriz $D=\left(X^{-1} Z+\gamma^{2} I\right)$ e a matriz $\left(A D A^{T}+\delta^{2} I\right)$ tornam-se mais mal-condicionadas, e o método demora para convergir. Nos métodos implementados, não pré-condicionamos a matriz A. Por isso, obtivemos grande número de iterações no Método dos Gradientes Conjugados.

\section{Conclusões}

Nesse trabalho apresentamos o problema de minimização Basis Pursuit, tendo como motivação sua aplicação em problemas envolvendo processamento de sinais.

Reescrevemos o problema Basis Pursuit como um problema de programação linear em sua forma padrão por meio de associações.

Consideramos a reformulação do problema como um problema quadrático e introduzimos o método Primal-Dual Barreira Logarítmica e nossos métodos propostos: Barreira Logarítmica e Primal-Dual Barreira Logarítmica Preditor-Corretor, sendo que este apresentou resultados satisfatórios em relação ao método implementado por Chen.

O valor da função objetivo foi muito similar para os métodos Primal-Dual Barreira Logarítmica e Primal-Dual Barreira Logarítmica Preditor-Corretor. O método Barreira Logarítmica não obteve resultados satisfatórios comparado aos outros métodos. Isso já era esperado, visto que não estamos considerando a condição de complementaridade.

Em relação ao tempo de convergência, também obtivemos tempos similares, com diferenças significativas em apenas dois sinais para os Métodos Primal-Dual Barreira Logarítmica e PrimalDual Barreira Logarítmica Preditor-Corretor. Para o método Barreira Logarítmica, quando seu 
tempo de convergência foi menor em comparação aos outros métodos, o valor da função objetivo não foi satisfatório.

O número de iterações realizadas pelo métodos Primal-Dual Barreira Logarítmica PreditorCorretor foi menor ou igual as realizadas pelo método Primal-Dual Barreira Logarítmica.

Portanto, obtivemos um melhor desempenho com as direções afim-escala, direção de centragem e direção de correção do Metodo Preditor Corretor, e um resultado menos eficiente quando não consideramos a condição de complementaridade.

\section{$5 \quad$ Agradecimentos}

Este trabalho foi parcialmente financiado pela FAPESP e CNPq.

\section{Referências}

[1] R. G. Baraniuk, Compressive sensing [lecture notes], Signal Processing Magazine, IEEE, 24, No. 4 (2007), 118-121.

[2] D. R. Cantane, E. G. Contharteze, A. R. L. Oliveira, Método de Pontos Interiores Barreira Logarítmica Preditor-Corretor Especializado para o Problema de Regressão pela Norma Lp, TEMA - Tend. Mat. Apl. Comput., 13, (2012), 219-231.

[3] S. S. Chen, "Basis Pursuit", Tese de Doutorado, Stanford University, 1995.

[4] S. S. Chen, D. L. Donoho, A. Michael, Atomic decomposition by basis pursuit, SIAM review, 43, No. 1 (2001), 129-159.

[5] S. S. Chen, D. L. Donoho, A. Michael, Atomizer for Matlab5. x, Disponível em: <http://www-stat. stanford. edu/ atomizer>, Acesso em: 15 nov. 2012.

[6] P. Gill, W. Murray, D. B. Ponceleon, A. Michael, Solving reduced KKT systems in barrier methods for linear and quadratic programming, DTIC Document, (1991).

[7] G. H. Golub, C. F. Van Loan, "Matrix computations", JHU Press, 2012.

[8] P. A. Kikuchi, "Métodos de Pontos Interiores Aplicados à Basis Pursuit", Dissertação de Mestrado, IMECC, Unicamp, Campinas, SP, 2013.

[9] I. J. Lustig, R. Marsten, D. F. Shanno, On implementing Mehrotra's predictor-corrector interior-point method for linear programming, SIAM Journal on Optimization, SIAM, 2, No. 3 (1992), 435-449.

[10] K. A. McShane, C. L. Monma, D. Shanno, An implementation of a primal-dual interior point method for linear programming, ORSA Journal on computing, INFORMS, 1, No. 2 (1989), 70-83.

[11] S. Mehrotra, On the implementation of a primal-dual interior point method, SIAM Journal on optimization, SIAM, 2, No. 4 (1992), 575-601.

[12] A. R. L. Oliveira, D. R. Cantane, Métodos de Pontos Interiores Aplicados ao Problema de Regressão pela Norma Lp, TEMA - Tend. Mat. Apl. Comput., 5, (2004), 269-279.

[13] S. J. Wright, "Primal-Dual Interior-Point Methods", em Society for Industrial and Applied Mathematics, Vol. 54, 1987. 